\section{Aspectos terapéuticos del covid-19: evidencia científica sobre uso de anticoagulantes en estos pacientes}

Therapeutic aspects of covid-19: scientific evidence on the use of anticoagulants in these patients

\section{Tarquino Adrián Pincay Rosales}

Md. Hospital General del Norte de

Guayaquil Los Ceibos,

thefather455@gmail.com,

https://orcid.org/0000-0001-6122-1411

Boris Xavier Caballero Pineda

Msc. Hospital Francisco Icaza Bustamante,

borisesc80@hotmail.com,

https://orcid.org/0000-0002-8843-7367

Manuel Roberto Parra Jiménez

Md. Centro de Salud Chinimbimi-Morona

Santiago, manuparra29@hotmail.com,

https://orcid.org/0000-0002-6473-8472

\section{Manuel Ricardo Chávez Salazar}

Md. Centro Materno Infantil Francisco

Jácome, ricardochavezs90@gmail.com,

https://orcid.org/0000-0003-3880-9422

Melissa Katyuska Marin Pineda

Md Hospital Abel Gilbert Ponton,

mdmedlissa_marin@hotmail.com

https://orcid.org/0000-0002-7003-0606

\section{Guayaquil - Ecuador}

http://www.jah-journal.com/index.php/jah

Journal of American health

Octubre - Diciembre vol. 3. Num. 3-2020

Esta obra está bajo una Licencia Creative Commons

Atribución-NoComercial-CompartirIgual 4.0 Internacional.

RECIBIDO: 8 DE MARZO 2019

ACEPTADO: 18 DE NOVIEMBRE 2019

PUBLICADO: 4 DE OCTUBRE 2020

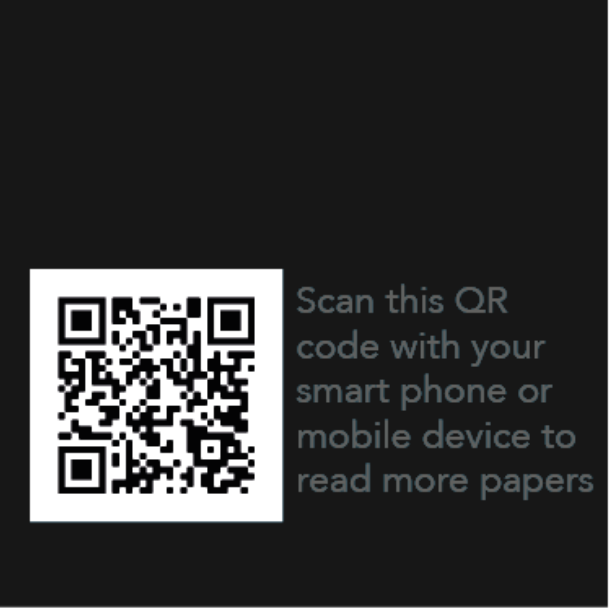

\section{RESUMEN}

Actualmente existen recomendaciones en cuanto a los enfoques terapéuticos en el caso de los anticoagulantes, dado los resultados positivos en varios hospitales en investigaciones experimentales. Sin embargo, existe aún limitaciones sobre la patogenia de la enfermedad, muchos de procesos fisiopatológicos aún no tienen explicación, por lo tanto, el control y tratamiento se complican en casos graves de pacientes que presentan factores de riesgos, no obstante ya es conocido el COVID-19 y su severidad en adultos mayores con presencia de enfermedades crónicas se asocian a una alta morbimortalidad. Uno de esos procesos fisiopatológicos son las alteraciones cardiovascular, la tromboembolia que se da como consecuencia de la activación de los procesos de coagulación que aún no se ha podido determinar porque sucede. Se pretende buscar y analizar la información publicada durante el 2020 y precisa respecto a la terapéutica de Covid-19 con énfasis en anticoagulantes en pacientes covid-19. Algunas investigaciones refieren que existe una relación entre anticoagulantes y con una mejor supervivencia hospitalaria entre los pacientes con COVID-19, tanto dentro como fuera de la unidad de cuidados intensivos, los pacientes que recibieron anticoagulantes tenían marcadores inflamatorios más altos en comparación con los pacientes no tratados con anticoagulantes. Demuestra que los anticoagulantes tomados por vía oral, subcutánea o intravenosa pueden desempeñar un papel importante en el cuidado de los pacientes con COVID-19, y estos pueden prevenir posibles eventos mortales asociados con el coronavirus, incluidos ataque cardíaco, accidente 
cerebrovascular y embolia pulmonar, pero, cada caso debe ser evaluado de forma individual, considerando los riesgosbeneficios, es importante seguir evaluando la efectividad de la terapia antitrombótica: antitrombótica oral, heparina subcutánea y heparina intravenosa a través de ensayos clínicos prospectivos.

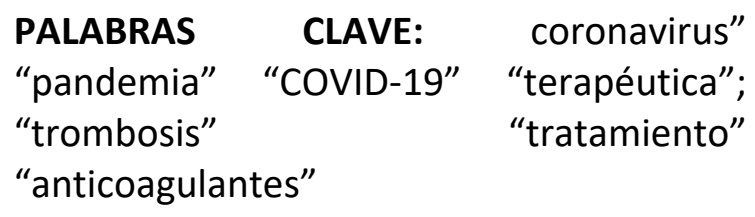

\section{ABSTRACT}

Currently there are recommendations regarding therapeutic approaches in the case of anticoagulants, given the positive results in several hospitals in experimental investigations. However, there are still limitations on the pathogenesis of the disease, many of the pathophysiological processes still have no explanation, therefore, the control and treatment are complicated in severe cases of patients with risk factors, and COVID-19 is already known and its severity in older adults with the presence of chronic diseases is associated with high morbidity and mortality. Coronavirus cases are increasing unevenly around the world. One of these pathophysiological processes is cardiovascular, the thromboembolism that occurs as a consequence of the activation of the coagulation processes that has not yet been determined why it happens. Some research reports that there is a relationship between anticoagulants and with better hospital survival among patients with COVID-19, both inside and outside the intensive care unit, patients who received anticoagulants had higher inflammatory markers compared to non-patients. treated with anticoagulants. Demonstrates that anticoagulants taken orally, subcutaneously or intravenously can play an important role in the care of patients with COVID-19, and these can prevent potential fatal events associated with the coronavirus, including heart attack, stroke, and pulmonary embolism, Despite each case being evaluated individually, considering the risks-benefits, it is important to continue evaluating the effectiveness of antithrombotic therapy: oral antithrombotic, subcutaneous heparin and intravenous heparin through prospective clinical trials.

KEYWORDS: coronavirus "“ pandemic "“ COVID-19 "“ therapeutic "; "Thrombosis" "treatment" "anticoagulants"

\section{INTRODUCCIÓN}

La pandemia de COVID-19 ha desencadenado la búsqueda vertiginosa de nuevas alternativas terapéuticas o preventivas que permitan mitigar su impacto, motivando la ejecución de cientos de ensayos clínicos destinados a evaluar su seguridad y eficacia. Algunos son agentes con actividad viral específica, otros son fármacos con indicaciones diferentes en los que se explora su eventual "reposicionamiento" o bien la búsqueda de estrategias para la inmunización activa o pasiva. (1) 
En ese contexto, las agencias regulatorias, los organismos sanitarios internacionales y las sociedades científicas en todo el mundo han emitido diferentes recomendaciones que orientan sobre las indicaciones y formas de administración de estas terapias. (1)

Existe evidencia una importante implicación de la hemostasia en la progresión de la enfermedad. Donde el síndrome de distress respiratorio, y la aparición de complicaciones trombóticas son formas graves, que obligan al personal médico a la adopción de medidas de manejo. (2). La respuesta inflamatoria que provoca el coronavirus favorece la aparición de trombosis venosa o arterial, y en fase avanzada aumenta el riesgo de coagulación intravascular diseminada, así como lesiones cardíacas (2), exhibiendo parámetros de coagulación de la sangre anormal o niveles de D-dímero muy elevados por lo que los anticoagulantes (AC) pueden mejorar la supervivencia de los pacientes con Covid-19, al prevenir infartos o ictus. (3), por lo que las estrategias de prevención efectiva con (AC) de la ETEV son cruciales (4)

La actividad inflamatoria propia de esta patología puede determinar una situación procoagulante, con implicación de múltiples mediadores, que se ha documentado en casos de sepsis. (5), es por esto que estos pacientes presentan un riesgo particularmente elevado de desarrollo de enfermedad tromboembólica venosa (ETEV), siendo necesario que se evalúe éste en todos los casos.

Por diversos análisis se concluyó que los tres factores de riesgo independientes de mortalidad a los 28 días suponian el aumento de diemro de (DD), el alargamiento del tiempo de protrombina y la disminución del recuento plaquetario (6).

El fervor desenfrenado por establecer la fisiopatología, un mecanismo causal que nos lleve a una estrategia terapéutica acertada ha llevado a la ciencia a buscar caminos conocidos y otros nunca transitados. Uno de ellos es la ya conocida vía común de la inflamación y coagulación con activación de un estado de hipercoagulabilidad. (7)

Aun así, existe limitada evidencia con relación a varios aspectos de la enfermedad (8). Diferentes publicaciones, proponen la implementación de diferentes pautas de anticoagulación empírica o tromboprofilaxis con dosis altas de heparina de bajo peso molecular. (8)

Recientes publicaciones han mostrado una alta tasa de eventos tromboembólicos, arteriales y venosos, que podrían explicar la morbimortalidad en algunos pacientes críticos con COVID-19. Lo anterior ha llevado a proponer intervenciones farmacológicas de antinflamatorios y anticoagulación. Sin embargo, es necesaria una juiciosa evaluación de los riesgos contrastados con los beneficios. (7).

Es importante destacar que algunas de estas alteraciones se han relacionado con un peor pronóstico, sobre todo el incremento del DD. Así, los pacientes con infección COVID 19 grave, especialmente los ingresados en Unidades de Cuidados Críticos, presentan un riesgo particularmente elevado de desarrollo de complicaciones cardiovasculares, en particular enfermedad tromboembólica venosa. En estas circunstancias, es importante establecer la terapia anticoagulante profiláctica o terapéutica que pueda beneficiar a estos pacientes. (5) Por ello, se puede decir que estos pacientes presentan un riesgo particularmente elevado de desarrollo de enfermedad tromboembólica venosa (ETEV), siendo necesario que se evalúe éste en todos los casos. Las escasas referencias publicadas hasta el momento sugieren que la tromboprofilaxis con heparina de bajo peso molecular (HBPM) puede ser beneficiosa en estos pacientes (5). En virtud de lo expuesto se pretende buscar y analizar la información publicada 
durante el 2020 y precisa respecto a la terapéutica de Covid-19 con énfasis de anticoagulantes en pacientes covid-19.

\section{MATERIALES Y MÉTODOS}

Este estudio de revisión y análisis terapéutico de pacientes con Covid-19, con énfasis en anticoagulantes se basó en una búsqueda exhaustiva de datos secundarios y terciarios en las bases literarias. Se emplearon buscadores como "coronavirus" y "pandemia" o "COVID-19" e "terapéutica"; "trombosis" and "tratamiento" or "anticoagulantes". Se analizaron todos los estudios publicados entre enero a septiembre 2020, publicados en español, inglés. Entre los buscadores literarios empleados están: Google académico, PubMed, Scopus, Scielo, Up to date, Latindex, y Web of Science, considerando 31 artículos relevantes de acuerdo al tema.

\section{RESULTADOS}

\section{Factores de Riesgo}

Es sabido ya, que el periodo de incubación de este virus oscila entre 2 y 14 días después de la exposición, con una presentación clínica muy variada, desde pacientes asintomáticos, pasando por casos leves, hasta pacientes críticamente enfermos con SDRA, microtrombosis, coagulación intravascular diseminada (CID), disfunción multiorgánica y muerte (7). La propagación de este virus hasta ahora ha sido global con mucha atención por parte de medios comunicativos. (9)

Se han confirmado más de 40 millones de casos de COVID-19 a nivel mundial, con un estimado de 4000.000 más de 700.000 pacientes recuperados, números que cambian día a día, y que pueden ser monitoreados en tiempo real en el sitio web de la Universidad Johns Hopkins, o con el Worldometer. (10), Toda la población es susceptible de infectarse, y el $80 \%$ de los casos sufrieron enfermedad leve, $14 \%$ enfermedad severa y el $5 \%$ fueron casos críticos que necesitaron UCI. (11); Sin embargo, un análisis de Scripps Research (Estados Unidos) hasta el 45\%, nunca muestra síntomas de la enfermedad, (12).

Las personas de cualquier edad y especialmente los adultos mayores $12 \%$ en mayores de 80 años (11), con afecciones concomitantes tienen mayor riesgo de enfermarse gravemente a causa del COVID-19 como cáncer, enfermedad renal cronica, Epoc, inmunodeprimidos por trasplante de órganos sólidos, Obesidad, afeccionescardiaca, enfermedad de células falciformes, Diabetes mellitus tipo 2,y profesionales de la salud. Con respecto a los médicos, se han visto altas tasas de mortalidad en especialidades como otorrinolaringología, anestesiología, neumología y odontología. (11) . (13)

La expansión del coronavirus ha seguido avanzando en Sudamérica, en donde 12 países se acercan a los cinco millones de contagiados y a 160000 fallecimientos. A nivel mundial al 8 de septiembre de 2020, se ha notificado a la Organización Mundial de la Salud (OMS) 27236916 casos confirmados, incluyendo 891031 muertes. (14)

\section{Clínica}

En relación al COVID-19, las manifestaciones clínicas comunes, encontradas en varios estudios, incluyen fiebre, tos predominantemente seca, fatiga/astenia, tos con expectoración, disnea, odinofagia y cefalea; además, una parte de los pacientes han manifestado síntomas 
gastrointestinales, como diarrea y vómitos, así como rinorrea, dolores musculares, confusión mental, anosmia y ageusia (15).

En los hombres hay una mayor frecuencia de fiebre, disnea, neumonía, síndrome de distrés respiratorio agudo y otros síntomas respiratorios. En las mujeres hay una mayor frecuencia de dolor de garganta, vómitos y diarrea puede inducir a una menor sospecha diagnóstica de esta enfermedad en las mujeres. (16)

La fiebre y la disnea son síntomas menos frecuentes y no sería adecuado tomarlos como criterio diagnóstico o de gravedad, en su lugar se proponen considerar datos objetivos, como saturación de oxígeno capilar o escores clínicos de fácil acceso para la atención inicial, y tomar acciones en el paciente. (17)

Por otra parte, los pacientes, una vez que están mejorando de la insuficiencia respiratoria, pueden sufrir una segunda oleada de complicaciones trombóticas (TEP, ictus, arteriales) a partir de los 10-15 días del ingreso que pueden causar el fallecimiento o graves secuelas. (18).

Hasta la actualidad no hay consenso sobre la causa de los coágulos en pacientes con covid-19. Se puede aseverar que son múltiples factores que deben estar presentes para que la sangre coagule y que el cuerpo busca constantemente un equilibrio entre la coagulación y el sangrado. Además, la inmovilización de muchos pacientes con Covid-19 es un factor que aumenta el riesgo de coagulación. (19).

Se ha reportado un porcentaje importante de pacientes que pueden presentar múltiples episodios trombóticos, isquemia periférica hasta tromboembolismo pulmonar y CID, relacionados a una respuesta macrofagia descontrolada, un estado de hipercoagubilidad y activación de la trombina. (19), y exhibir una situación procoagulante, con implicación de múltiples mediadores, que se ha documentado en casos de sepsis. (20)

Existe un I patrón típico coagulante que incluye las siguientes características: Incremento significativo en el nivel plasmático del dímero $D$, incremento significativo en el nivel plasmático de fibrinógeno, alargamiento moderado del tiempo de protrombina, normalidad en el tiempo parcial de tromboplastina activada, tendencia a trombocitosis moderada y disminución moderada, no clínicamente significativa, de la actividad de antitrombina. (4)

Una de las principales complicaciones que dificulta el manejo de los pacientes con COVID-19 es la formación de trombos o coágulos de sangre que pueden provocar eventos tromboembólicos potencialmente mortales. Los anticoagulantes pueden mejorar la supervivencia de los pacientes hospitalizados con COVID-19 al prevenir posibles eventos mortales asociados con el coronavirus, como infartos o ictus.(6)

El aumento de DD se ha visto relacionado, no sólo con mayor gravedad clínica (deterioro de la insuficiencia respiratoria) sino también con un aumento de la vascularización pulmonar y el desarrollo de microtrombos en su interior. (6)

Los trastornos hemostáticos descriptos en ese contexto son la expresión de un estado de hipercoagulabilidad desencadenado por múltiples factores entre los que se destacan la inmovilidad, la enfermedad crítica y la inflamación, entre otros.(3) 
Se han utilizado varios medicamentos antivirales; entre ellos, ribavirina, la combinación de lopinavir/ritonavir y remdesivir. Sin embargo, se deben esperar los resultados que arrojen los múltiples ensayos clínicos que se están llevando a cabo, antes de poderse determinar una terapia antiviral efectiva. (13). La evidencia in vivo es muy limitada, ensayos pequeños determinan que el tratamiento con Fosfato de Cloroquina produjo "mejoría significativa" en pacientes con neumonía, se considera que la hidroxicloroquina es menos toxica que Cloroquina. (21). Sin embargo, el riesgo de arritmias cardíacas debe considerarse. (21).

Luego tenemos los otros fármacos encaminados a detener la inflamación, acción procoagulante, el tratamiento del shock.

\section{DISCUSIÓN}

Hasta el momento no hay un tratamiento antiviral específico aprobado por la FDA. Los pacientes con cuadros leves deben ser manejados sintomáticamente y aislados en su casa. Los casos graves son aislados en los centros de atención, y el tratamiento es enfocado principalmente al alivio de los síntomas generales, la oxigenoterapia y, en los casos críticos, al soporte respiratorio, con o sin ventilación mecánica. (13). La terapia con oxígeno se reserva para casos severos de la enfermedad con el objetivo de mantener la saturación de oxígeno $>90 \%$. (11). El ventilador mecánico se utiliza en casos de falla ventilatoria refractaria a oxigenoterapia. A pesar de no contar con un tratamiento antiviral especifico, se han propuesto medicamentos como Remdesivir, Lopinavir/Ritonavir, así como otros medicamentos como Cloroquina, Hidroxicloroquina e Interferón-alfa y beta. (11), (21), Otro aspecto es el soporte preventivo, mediante recomendaciones de aislamiento respiratorio y de contactos.

La FDA está investigando una controversia que ha surgido con respecto al uso de AINE en pacientes con COVID-19; sin embargo, no hay evidencia publicada que relacione el uso de AINE con el empeoramiento de los síntomas de COVID-19. Hasta que se disponga de datos adicionales, se puede preferir el acetaminofén para el control de la temperatura. (21)

El tratamiento del choque séptico incluye el uso de vasopresores si la administración de líquidos no restablece la perfusión adecuada. En adultos, está indicada noradrenalina. La epinefrina o la vasopresina se prefieren como segunda línea; la dopamina si no están disponibles las anteriores. La meta es lograr, presión arterial media de $65 \mathrm{~mm} \mathrm{Hg}$ o más; en adultos mayores 60 a $65 \mathrm{~mm} \mathrm{Hg.} \mathrm{(21)}$

La respuesta inflamatoria que provoca el coronavirus favorece la aparición de trombosis venosa o arterial, lesiones cardíacas, por lo que los anticoagulantes pueden mejorar la supervivencia de los pacientes, al prevenir infartos o ictus. (22) lo que hace que pacientes con COVID-19 tienen un mayor riesgo de padecer una enfermedad tromboembólica venosa, debido a que los pacientes presentan parámetros de coagulación de la sangre anormales o tienen niveles de D-dímero muy elevados (22)

Un desafío terapéutico son los pacientes que desarrollan trombocitopenia inducida por heparinas (HIT). Para ellos, el consenso sugiere argatrobán o bivalirudina, anticoagulantes parenterales, sin mención de anticoagulantes orales. (25)

Los anticoagulantes como la heparina podría detener hasta en un 70 \% la entrada del COVID19 en las células, según un estudio realizado por la Universidad Federal de Sao Paulo (Unifesp), 
con la colaboración de científicos ingleses e italianos (23), además de combatir los trastornos de la coagulación que pueden afectar los vasos pulmonares y la oxigenación, parece tener la capacidad de "dificultar" la entrada del SARS-CoV-2 en las células. (23). Los científicos realizaron pruebas de laboratorio en linajes celulares provenientes de riñón de mono verde africano (Cercopithecus aethiops) y comprobaron que la heparina redujo la invasión de células por el nuevo coronavirus en un 70 \%. (23) . Los experimentos confirmaron que cuando la heparina se une a las proteínas de la punta del SARS-CoV-2, ocasiona en esas moléculas una alteración conformacional que llevaría a una especie de «bloqueo» para el virus. (24)

La heparina (heparina sódica y enoxaparina) se encuentra en la cúspide del manejo de la coagulopatía inducida por COVID-19. Propone realizar tromboprofilaxis a todos los pacientes sospechosos y confirmados para el virus, que estén asintomáticos o con síntomas leves, con revaluación periódica del estado de coagulación. (25)

Para los pacientes severos con elevado riesgo de coagulación intravascular diseminada o coagulopatía inducida por sepsis, el consenso recomienda anticoagulación con heparina sódica $1 \mathrm{Ul} / \mathrm{kg} / \mathrm{hr}$ por vía endovenosa o bien, enoxaparina $1 \mathrm{mg} / \mathrm{kg}$ cada 12 horas por vía subcutánea, para el paciente moderado. (25)

La heparina no fraccionada, la heparina de bajo peso molecular y la warfarina no se acumulan en la leche humana y no producen un efecto anticoagulante en el recién nacido. Por lo tanto, estos fármacos se pueden administrar en las mujeres lactantes, ya presenten COVID-19 o no, si resultara imprescindible prevenir o tratar la ETV (A III). (26)

Para pacientes ambulatorios con tratamiento anticoagulante oral previo, o se recomienda cambios de tratamiento en los pacientes ya anticoagulados con anticoagulantes orales de acción directa (ACOD) o con antagonistas de la vitamina K (AVK), estables y con un buen control de INR. (27)

Tras el alta hospitalaria, pacientes con Sintrom ${ }^{\circledR}$ previo: se recomienda mantener la HBPM durante dos semanas y contactar con Hematología para el reinicio. Pacientes con ACOD previo: se recomienda reiniciarlo si no hay interacciones farmacológicas con el tratamiento al alta. (27)

Toda actuación en la consulta de anticoagulación se hará con la intención de que no se generen visitas sucesivas en los siguientes 2 meses, para lo cual se propone que los pacientes con fibrilación auricular no valvular tratados con fármacos antivitamina $\mathrm{K}$ que puedan requerir visitas cercanas por incertidumbre en la dosis (lo que incluye los inicios) y que no tengan contraindicación, se valorará pasarlos a anticoagulantes orales de acción directa. (28)

Para ello se precisa que las inspecciones médicas autoricen el uso financiado del fármaco con una posterior revisión a los 4-6 meses, cuando lo permita la situación de alto riesgo por la movilidad de pacientes y sanitarios (28)

Se intentará prolongar lo más posible el control de los fármacos antivitamina $\mathrm{K}$ en los pacientes bien controlados, llegando a controles cada 6-8 semanas si el hematólogo o su médico de atención primaria lo valora indicado. Se intentará, en la medida de lo posible, ampliar las horas en que se realizan las determinaciones analíticas de INR para evitar las aglomeraciones. (28) 
Para el tratamiento con anticoagulante las indicaciones de anticoagulación crónica son bien conocidas, e incluyen fibrilación auricular, ictus, antecedentes recientes de ETEV o portadores de válvulas cardíacas mecánicas (12). En los pacientes con infección por COVID-19 que estuvieran en tratamiento con un fármaco anticoagulante, ya sea del tipo antivitamina $\mathrm{k} o$ anticoagulante oral directo, se debe mantener la anticoagulación independientemente del curso de la enfermedad, siempre que no se asocien eventos hemorrágicos. (5)

Se han descubierto que una de las complicaciones más preocupantes del Covid 19, es la inusual formación de coágulos sanguíneos en numerosos pacientes, incluso en aquellos que se encontraban recibiendo anticoagulantes. Estos coágulos pueden llegar a órganos como el pulmón, el corazón o el cerebro, y causar ataques cardíacos o accidentes cerebrovasculares.(12)

Los anticoagulantes tomados por vía oral, subcutánea o intravenosa pueden desempeñar un papel importante en el cuidado de los pacientes con Covid-19 y estos pueden prevenir posibles eventos mortales asociados con el coronavirus.(2)

Investigadores del Instituto Politécnico Rensselaer han comprobado que la heparina, puede ser eficaz para combatir el Covid-19, este virus utiliza una proteína de pico de superficie para adherirse a las células humanas e iniciar la infección. En este sentido, se ha comprobado que la heparina, se une fuertemente con la proteína de la punta de la superficie, bloqueando potencialmente la infección.(13)

El consenso chino sobre manejo de la coagulación en COVID-19, coloca a las heparinas en la cúspide del manejo de la coagulapatía inducida por COVID-19. Propone realizar tromboprofilaxis a todos los pacientes sospechosos y confirmados para el virus, que estén asintomáticos o con síntomas leves, con revaluación periódica del estado de coagulación. (14)

En casos hospitalizados con $\mathrm{CIC}-19$, de alto riesgo y/o elevación en los niveles de dímero-D se recomienda el uso de HBPM en dosis de profilaxis intermedia (enoxaparina $40 \mathrm{mg}$ cada $12 \mathrm{~h}$ ) o HNF sódica (12 U/kg/hora) con el objetivo de controlar la microtrombosis y disminuir la incidencia de TEV. Se recomiendo lo mismo para pacientes internados con infección severa (15)

Recientes casos informan de un $25 \%$ y un $27 \%$ de casos de tromboembolismo venoso con neumonía grave por COVID-19, que se prefiere la administración de la heparina de bajo peso molecular a la heparina no fraccionada para reducir el contacto con el paciente; fondaparinux se recomienda en pacientes con antecedentes de trombocitopenia inducida por heparina. (16)

Se puede administrar anticoagulación plena con heparina no fraccionada o con HBPM, Las heparinas son sustancias anticoagulantes inyectables y se usan cuando se requiere una acción anticoagulante rápida y de corta duración.(17)

El uso de HBPM reduce la generación de trombina y la aparición de un evento tromboembólico venoso, es conocido que tiene propiedades antiinflamatorias, lo cual puede ayudar en el control de esta enfermedad, en la que se produce un marcado aumento de citocinas proinflamatorias, estudios de pacientes críticos, el uso de HBPM disminuyó la afección inflamatoria.(4) 
Si la anticoagulación está contraindicada, los pacientes deben tener profilaxis mecánica, Actualmente un incremento en el Dímero-D NO es indicación para escalar la terapia de anticoagulación, Pacientes ingresados al $\mathrm{MGH}$ quienes se encuentren recibiendo anticoagulantes de rutina y tienen bajo riesgo de COVID-19 pueden mantener su tratamiento anticoagulante.(18)

Los anticoagulantes tomados por vía oral, subcutánea o intravenosa pueden desempeñar un papel importante en el cuidado de los pacientes con COVID-19, y estos pueden prevenir posibles eventos mortales asociados con el coronavirus, incluidos ataque cardíaco, accidente cerebrovascular y embolia pulmonar. (29)

En los pacientes que requieren iniciar (por primera vez) anticoagulación durante el período de alarma Sanitaria existen opciones para cardiopatías, enfermedades trombóticas venosa, coagulopatía por covid-19.

\section{CONCLUSIONES}

El covid-19 continúa siendo un problema de salud pública por los gastos que genera, el grupo de personas a las que afecta y por la búsqueda constate de nuevos y efectivos fármacos o vacunas para tratar y prevenir esta enfermedad. Los primeros informes indican la utilización empírica de varios tratamientos farmacológicos, estos tratamientos incluyen antivirales que pueden bloquear la unión viral, inhibir la producción de ARN viral o inhiben la liberación de partículas de virión maduras. Otros fármacos como los inmunosupresores son destinados a mitigar las reacciones de tipo autoinmune involucradas en el síndrome de dificultad respiratoria aguda (SDRA) con neumonitis. Recientemente, se han demostrado una incidencia preocupante de coagulopatía viral, por lo que la anticoagulación terapéutica es una opción sobre todo se reserva para los diagnósticos confirmados de ETEV. Sin embargo, algunos clínicos han planteado el uso de anticoagulación terapéutica en los pacientes críticos, aún en ausencia de confirmación de ETEV, bajo la hipótesis de un potencial beneficio para prevenir la trombosis microvascular. La heparina de bajo peso molecular ajustada al peso del paciente con Covid-19, entre otros como la enoxaparina, tinzaparina, beiparina, nadroparina, dalteparina son algunos fármacos disponibles para estos casos.

\section{REFERENCIAS}

1. Garrahan HdPJP. RECOMENDACIONES DE ORGANISMOS, AGENCIAS DE SALUD Y SOCIEDADES PARA EL TRATAMIENTO FARMACOLOGICO DE COVID-19. Elsevier. 2020 Julio.

2. Sevilla HUVdR. Junta de Andalucía. Consejería de Salud y Familias. [Online].; 2020. Available from:

https://www.google.com/url?sa=t\&rct=i\&q=\&esrc=s\&source=web\&cd=\&cad=ria\&uact=8\&ve d=2ahUKEwjx pbnh9vrAhXCjVkKHVZMA70QFjAAegQIBBAB\&url=https\%3A\%2F\%2Fwww.seme sandalucia.es\%2Fwp-content\%2Fuploads\%2F2020\%2F04\%2FProtocolo-Hemostasia-COVID19 v1 1.pdf\&usg=AOvVaw1.

3. Vecilla J BECJMM. Anticoagulación en COVID. Recimundo. 2020 Jul 01;: p. 23-41. 
4. Vivas D RVEPMRITMA, Ruiz-Nodar JM CSJMGJ, Consuegra L LFJMFA, V AMCAPVJ.

Recomendaciones sobre el tratamiento antitrombotico durante la pandemia COVID-19.

[Online].; 2020. Available from: https://doi.org/doi:10.1016/j.recesp.2020.04.006.

5. Juan V RFPSFHCC, G A. Propuesta de recomendaciones de manejo de farmacos anticoagulantes y antiagregantes en los pacientes graves con infeccion por Covid-19. Scielo. 2020 Junio.

6. Llau V FRSPHFCCGA. Propuesta de recomendaciones de manejo de fármacos anticoagulantes y antiagregantes en los pacientes graves con infección por COVID-19. La Paz, Madrid. :; 2020.

7. Abuabara E, Serpa D, Leal V, Pajaro N, Correa J, Rico J. Salud UIS. [Online].; 2020. Available from:

https://www.researchgate.net/profile/Jose Bohorquez Rivero2/publication/343808961 Anti coagulation in patients with SARS-CoV-2 InfectionCOVID-

19/links/5f4097fc92851cd3021473ce/Anticoagulation-in-patients-with-SARS-CoV-2-InfectionCOVID-19.pdf.

8. Moreno G, Carbonell R, Bodi A, Rodriguez A. Science Direct. [Online].; 2020. Available from: https://www.sciencedirect.com/science/article/pii/S021056912030187X.

9. Sardi A. COVID-19 trombosis y hemoglobina. ISAEM. 2020 Mayo.

10. Salud OMdl. Actualizacion Epidemiologica Nuevo Coronavirus. Scielo. 2020 Febrero.

11. Otoya A, Garcia M, Jaramillo C, Wills C, Campos A. Acta de Otorrinolaringologia \& Cirugia de Cabeza y Cuello. [Online].; 2020. Available from:

http://docs.bvsalud.org/biblioref/2020/05/1096161/1-covid-19-generalidadescomportamiento-epidemiologico.pdf.

12. Infosalus. [Online].; 2020. Available from: https://www.infosalus.com/actualidad/noticia-45contagios-covid-19-pueden-ser-asintomaticos-20200615124632.html.

13. Francisco Diaz AT. SARS-CoV-2/COVID-19: el virus, la enfermedad y la pandemia. Elsevier. 2020 Abril.

14. World Health Organization. [Online].; 2020. Available from: https://covid19.who.int/.

15. CorSalud. Repercusión cardiovascular de la infección por el nuevo coronavirus. Sociedad Cubana de Cardiología. 2020 Sep; 12(1).

16. Canteroa M. ELSEVIER. [Online].; 2020. Available from: https://rua.ua.es/dspace/bitstream/10045/108364/1/Ruiz-Cantero 2020 GacSanit.pdf.

17. Acosta G, Escobar G, Bernaola G, Alfaro J, Taype W, Marcos C. Revista Peruana de Medicina Experimental y Salud Publica. [Online].; 2020. Available from:

https://rpmesp.ins.gob.pe/index.php/rpmesp/article/view/5437/3535. 
18. Junta de Andalucía. Concejería de Salud de Andalucía. [Online]. [cited 2020 Septiembre 07. Available from: https://www.semesandalucia.es/wp-content/uploads/2020/04/ProtocoloHemostasia-COVID-19 v1 1.pdf.

19. Paredes C, Parra C, Carranza K, Mayorga L. Revista Reciamuc. [Online].; 2020. Available from: https://reciamuc.com/index.php/RECIAMUC/article/view/498/758.

20. Llau J, Ferrandis R, Sierra P, Hidalgo F, Cassinello C, Gomez A. SEDAR. [Online].; 2020. Available from:

https://www.sedar.es/images/site/NOTICIAS/coronavirus/RECOMENDACIONES hemostasiaCOVID-final.pdf.

21. Herrera D, Gaus D. [Online].; 2020. Available from: https://doi.org/10.23936/pfr.v5i1.146.

22. Vecilla J, Barco E, Carvajal J, Salome M. Revista Cientifica Mundo de la Investigacion y el Conocimiento. [Online].; 2020. Available from:

http://recimundo.com/index.php/es/article/view/846.

23. ILAPHAR. [Online].; 2020. Available from: https://www.revistadelaofil.org/covid-19-el-turnode-los-anticoagulantes/.

24. EL MERCURIO. [Online].; 2020. Available from: https://ww2.elmercurio.com.ec/2020/05/11/identifican-anticoagulante-que-podria-frenarentrada-de-covid-19-en-celulas/\#. 